Supporting Information:

\title{
Mechanisms of Pressure-Induced Structural Transformation in Confined Sodium Borates Glasses
}

\author{
Huong T. T. Ta ${ }^{1}$, A. Kiet Tieu ${ }^{1}$, Hongtao Zhu ${ }^{*}$, Haibo Yu ${ }^{2,3^{*}}$, Nam V. Tran ${ }^{1}$, Thi D. Ta ${ }^{1}$
}

${ }^{1}$ School of Mechanical, Materials, Mechatronic and Biomedical Engineering, University of Wollongong, Northfields Avenue, Wollongong, NSW 2522, Australia

${ }^{2}$ School of Chemistry and Molecular Bioscience, University of Wollongong, Northfields Avenue, Wollongong, NSW 2522, Australia

${ }^{3}$ Molecular Horizons, University of Wollongong, Northfields Avenue, Wollongong, NSW 2522, Australia

*Corresponding author: hongtao@uow.edu.au;

hyu@uow.edu.au 


\section{Structures of sodium borate glasses at $300 \mathrm{~K}$ after cooling}

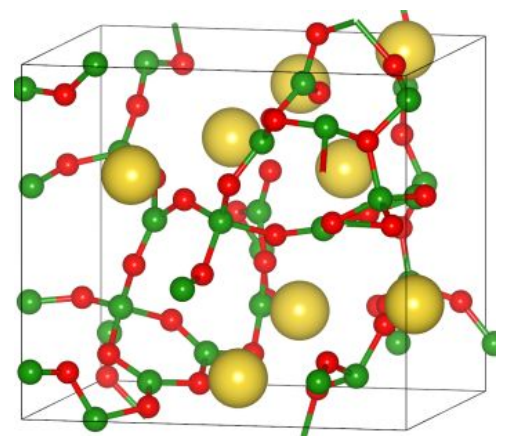

(a) $\mathrm{Na}_{2} \mathrm{O} \cdot 3 \mathrm{~B}_{2} \mathrm{O}_{3}$

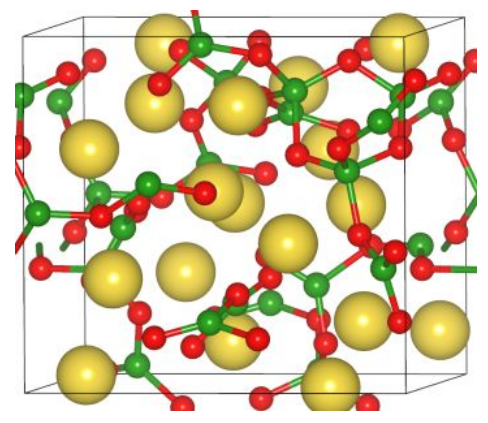

(c) $\mathrm{Na}_{2} \mathrm{O} \cdot 1 \mathrm{~B}_{2} \mathrm{O}_{3}$

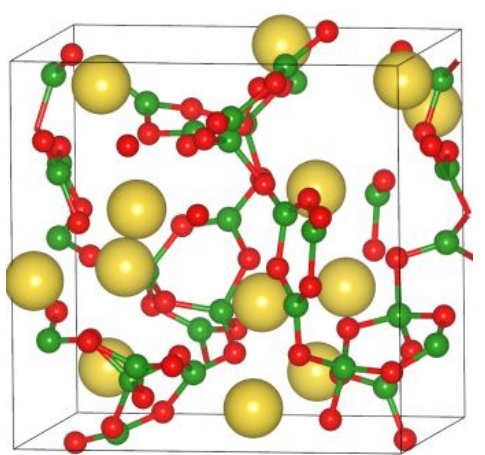

(b) $\mathrm{Na}_{2} \mathrm{O} \cdot 2 \mathrm{~B}_{2} \mathrm{O}_{3}$

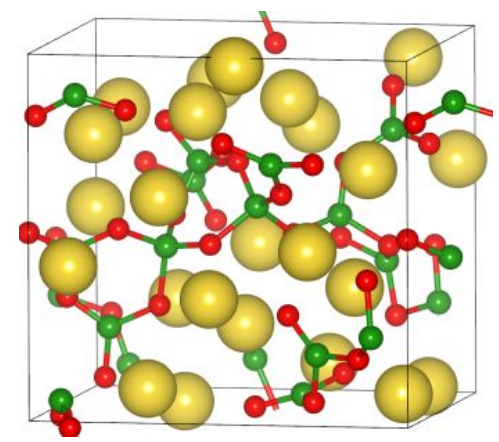

(d) $3 \mathrm{Na}_{2} \mathrm{O} \cdot 2 \mathrm{~B}_{2} \mathrm{O}_{3}$

Figure S1. Visualization structures of sodium borate glasses at $300 \mathrm{~K}$ obtained from cooling process by AIMD simulation.
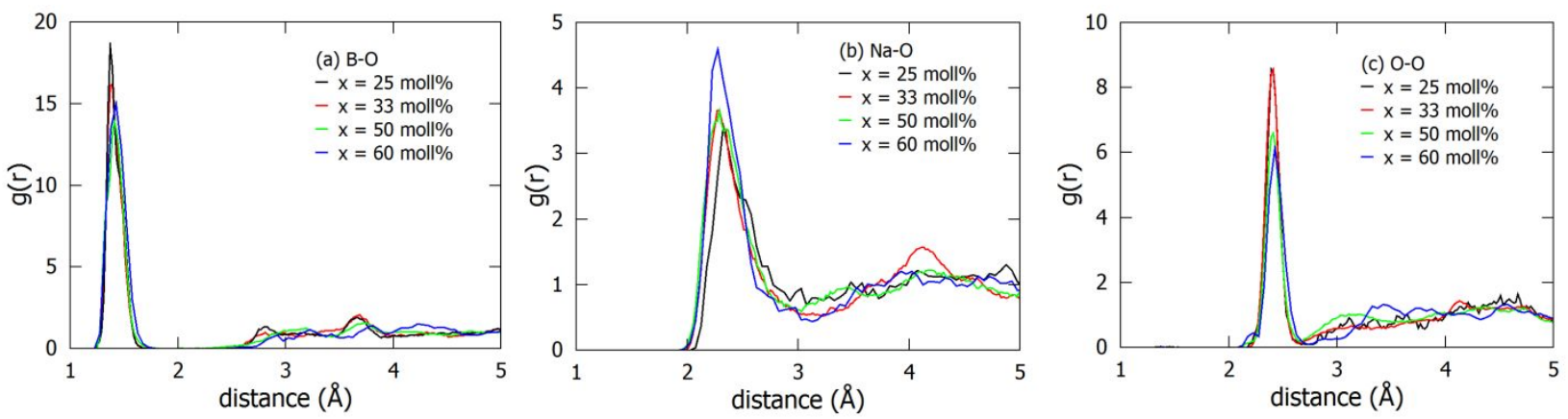

Figure S2. Radial distribution function $\mathrm{g}(\mathrm{r})$ of $\mathrm{B}-\mathrm{O}, \mathrm{Na}-\mathrm{O}$, and $\mathrm{O}-\mathrm{O}$ pairs in $x \mathrm{Na}_{2} \mathrm{O} \cdot(100-x) \mathrm{B}_{2} \mathrm{O}_{3}$ systems at $300 \mathrm{~K}$. The first peaks of $\mathrm{g}(\mathrm{r})$ correspond to the bond length of each pair presented in Table S1. 
Table S1. Structural parameters of $\mathrm{xNa}_{2} \mathrm{O} \cdot(100-\mathrm{x}) \mathrm{B}_{2} \mathrm{O}_{3}$ at $300 \mathrm{~K}$ compared with experimental data (where available).

\begin{tabular}{|c|c|c|c|c|c|c|c|c|c|}
\hline & \multicolumn{2}{|c|}{$\mathrm{B}^{4} / \mathrm{B}^{3}(\%)$} & \multicolumn{9}{|c|}{ bond length $(\AA)$} \\
\hline Glass & AIMD & Exp. ${ }^{1}$ & ${ }^{[3]} \mathrm{B}-\mathrm{O} /[4] \mathrm{B}-\mathrm{O}$ & Exp. $^{2}$ & Exp. $^{3}$ & $\mathrm{Na}-\mathrm{O}$ & Exp. $^{2}$ & $\mathrm{O}-\mathrm{O}$ & Exp. $^{4}$ \\
\hline $\begin{array}{c}\mathrm{Na}_{2} \mathrm{O} \cdot 3 \mathrm{~B}_{2} \mathrm{O}_{3} \\
\left(\mathrm{Na}_{2} \mathrm{O} 25 \%\right)\end{array}$ & 25.0 & 35 & $1.38 / 1.48$ & 1.416 & & 2.33 & 2.40 & 2.39 & \\
\hline $\begin{array}{c}\mathrm{Na}_{2} \mathrm{O} \cdot 2 \mathrm{~B}_{2} \mathrm{O}_{3} \\
\left(\mathrm{Na}_{2} \mathrm{O} 33 \%\right)\end{array}$ & 37.5 & 43 & $1.38 / 1.49$ & 1.436 & $1.38 / 1.485$ & 2.29 & 2.37 & 2.41 & $2.41(4)$ \\
\hline $\begin{array}{c}\mathrm{Na}_{2} \mathrm{O} \cdot 1 \mathrm{~B}_{2} \mathrm{O}_{3} \\
\left(\mathrm{Na}_{2} \mathrm{O} 50 \%\right)\end{array}$ & 33.3 & 34 & $1.39 / 1.48$ & & & 2.29 & & 2.41 & \\
\hline $\begin{array}{c}3 \mathrm{Na}_{2} \mathrm{O} \cdot 2 \mathrm{~B}_{2} \mathrm{O}_{3} \\
\left(\mathrm{Na}_{2} \mathrm{O} 60 \%\right)\end{array}$ & 18.8 & & $1.40 / 1.50$ & & & 2.28 & & 2.43 & \\
\hline
\end{tabular}

\section{Cooling rate effects on sodium borates structures at $300 \mathrm{~K}$}

In order to check the effect of cooling rate on the structures of the obtained sodium borate glasses at $300 \mathrm{~K}$, we tested the cooling process of $\mathrm{Na}_{2} \mathrm{O} \cdot 2 \mathrm{~B}_{2} \mathrm{O}_{3}$ with three different cooling rates: $5.0 \times 10^{13} \mathrm{~K} \cdot \mathrm{s}^{-1}, 1.0 \times 10^{13} \mathrm{~K} \cdot \mathrm{s}^{-1}, 5.0 \times 10^{12} \mathrm{~K} \cdot \mathrm{s}^{-1}$. The structural parameters of the glasses produced using three cooling rate are presented in Table S2.

Table S2. Structural parameters of $\mathrm{Na}_{2} \mathrm{O} \cdot 2 \mathrm{~B}_{2} \mathrm{O}_{3}$ at $300 \mathrm{~K}$ obtained from the cooling process with three different cooling rates.

\begin{tabular}{|c|c|c|c|c|c|}
\hline & $\begin{array}{c}\mathrm{B}^{4} / \mathrm{B}^{3} \\
(\%)\end{array}$ & ${ }^{[3]} \mathrm{B}-\mathrm{O}$ & ${ }^{[4]} \mathrm{B}-\mathrm{O}$ & $\mathrm{Na}-\mathrm{O}$ & $\mathrm{O}-\mathrm{O}$ \\
\hline Exp. & $43.0^{1}$ & $1.38^{3}$ & $1.485^{3}$ & $2.37^{2}$ & $2.41^{4}$ \\
\hline $\begin{array}{c}\text { Cooling rate } 1 \\
5.0 \times 10^{13} \mathrm{~K} \cdot \mathrm{s}^{-1}\end{array}$ & 41.6 & 1.38 & 1.492 & 2.33 & 2.43 \\
\hline $\begin{array}{c}\text { Cooling rate } 2 \\
1.0 \times 10^{13} \mathrm{~K} \cdot \mathrm{s}^{-1}\end{array}$ & 37.5 & 1.38 & 1.485 & 2.29 & 2.41 \\
\hline $\begin{array}{c}\text { Cooling rate } 3 \\
5.0 \times 10^{12} \mathrm{~K} \cdot \mathrm{s}^{-1}\end{array}$ & 37.5 & 1.38 & 1.486 & 2.33 & 2.43 \\
\hline
\end{tabular}


The results in table S2 show that the structural parameters of sodium borate glasses at $300 \mathrm{~K}$ obtained from three different cooling rates are similar, which indicates a minor effects of cooling rate on short- and medium-range structure of borate glasses. ${ }^{2}$ We used cooling rate 2 as it requires an affordable computer resource and comparable with that used in other AIMD work. ${ }^{5}$

\section{Changes of sodium borate band gaps}

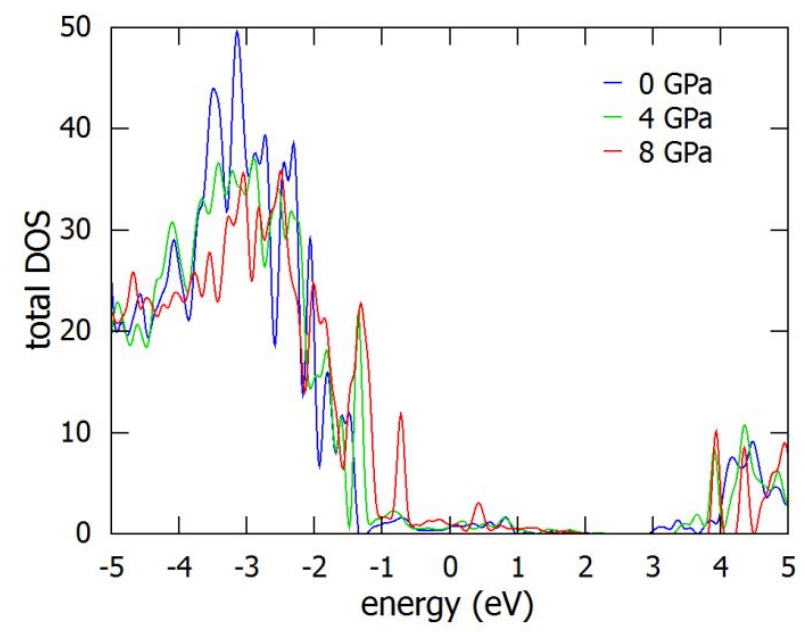

Figure S3. Total DOS of sodium borate $\mathrm{Na}_{2} \mathrm{O}_{2} 2 \mathrm{~B}_{2} \mathrm{O}_{3}$ at 0,4 and $8 \mathrm{GPa}$.

\section{References}

(1) Shibata, M.; Sanchez, C.; Patel, H.; Feller, S.; Stark, J.; Sumcad, G.; Kasper, J., The Density of Lithium Borate Glasses Related to Atomic Arrangements. J. Non-Cryst. Solids 1986, 85, 29-41.

(2) Ennas, G.; Musinu, A.; Piccaluga, G.; Pinna, G., Effect of Cooling Rate on the Structure of Sodium Borate Glasses. Zeitschrift für Naturforschung A, 1993, 48, 599 - 604.

(3) Majérus, O.; Cormier, L.; Calas, G.; Beuneu, B., Temperature-Induced Boron Coordination Change in Alkali Borate Glasses and Melts. Phys. Rev.B 2003, 67, 024210.

(4) Alderman, O. L. G.; Liška, M.; Macháček, J.; Benmore, C. J.; Lin, A.; Tamalonis, A.; Weber, J. K. R. Temperature-Driven Structural Transitions in Molten Sodium Borates $\mathrm{Na}_{2} \mathrm{O}-\mathrm{B}_{2} \mathrm{O}_{3}$ : X-Ray Diffraction, Thermodynamic Modeling, and Implications for Topological Constraint Theory. J. Phys. Chem. C 2016, 120, 553-560.

(5) Lei, X.; Jee, Y.; Huang, K., Amorphous $\mathrm{Na}_{2} \mathrm{Si}_{2} \mathrm{O}_{5}$ as a Fast $\mathrm{Na}^{+}$Conductor: An Ab Initio Molecular Dynamics Simulation. J. Mater. Chem. A 2015, 3, 19920-19927. 